\title{
ECUACIONES DIFERENCIALES Y DIFERENCIAS FINITAS
}

\section{DIFFERENTIAL EQUATIONS AND FINITE DIFFERENCE}

Arturo Pérez París: Universidad de Alcalá. Madrid (España).

arturo.perez@mixmail.com

Julio Gutiérrez Muñoz: Universidad de Alcalá. Madrid (España). julio.gutierrez@uah.es

\section{CURRÍCULUM VITAE}

Escuela Politécnica de la Universidad de Alcalá de Henares. Ingeniero electrónico y literato. Destacan sus estudios sobre aplicaciones eléctricas y motricidad sobre los que ha publicado varios artículos científicos.

Catedrático de Física Atómica, Molecular y Nuclear de la Escuela Politécnica Superior de la Universidad de Alcalá de Henares (Madrid). En la actualidad imparte clases de la asignatura de Física incluida en la titulación de Grado en Química de dicha Universidad. Autor de numerosos artículos científicos. Miembro fundador de GRUA (Grupo de Reflexión de la Universidad de Alcalá de Henares). Vicerrector de la Universidad de Alcalá de Henares. Director de la Revista Vivat Academia.

\section{RESUMEN}

En los tres artículos anteriores publicados hemos realizado una exposición sencilla de las propiedades generales de las ecuaciones en diferencias, así como de los 
métodos más simples empleados en su resolución. En este caso abordaremos el paralelismo entre la resolución de ecuaciones diferenciales y ecuaciones en diferencias. Se hará a partir del estudio de un caso particular muy simple de ecuación diferencial ordinaria de primer orden, no homogénea, con coeficientes constante. El método utilizado es el de las diferencias finitas.

\section{PALABRAS CLAVE}

Ecuaciones en diferencias - Resolución - Ecuaciones diferenciales - Diferencias finitas

\section{ABSTRACT}

In the three previous articles we have made a simple statement of the general properties of difference equations, and simple methods used in their resolution. In this case addresses the parallel between the resolution of differential equations and difference equations. Will be from a case study of simple ordinary differential equation of first order, homogeneous, with constant coefficients. The method used is finite differences.

\section{KEY WORDS}

Difference equations - Resolution - Differential equations - Finite difference

\section{ÍNDICE}

\section{Introducción}

2. Paralelismo en la resolución de ecuaciones diferenciales y ecuaciones en diferencias

3. Paralelismo funcional 
4. El método de las diferencias finitas

5. Conclusión

6. Bibliografía

\section{TEXTO}

\section{Introducción}

En los tres artículos anteriores publicados hemos realizado una exposición sencilla de las propiedades generales de las ecuaciones en diferencias, así como de los métodos más simples empleados en su resolución. Comenzamos por presentar el método estándar de la recurrencia, como criterio general de encontrar soluciones a este tipo de problemas, propios de muchas áreas de la ciencia y la tecnología (Física, Biología, Economía, Ingeniería, etc.), y ligados a la estabilidad, asintoticidad de un comportamiento dinámico, oscilaciones, teoría del control, caos, fractales, etc. (“Torres de Hanoi”. Vivat Academia $n^{\circ}$ 30, noviembre 2001). Posteriormente (Ecuaciones en Diferencias: Planteamiento general. Vivat Academia $n^{\circ}$ 31, diciembre 2001- enero 2002) procedimos a su estudio somero y general, dando los criterios de estabilidad y asintoticidad, así como una serie de pautas para poder manipular adecuadamente las ecuaciones lineales en diferencias.

El método de la recurrencia puede ser muy útil en los casos más simples, sin embargo, en la mayoría de los planteamientos debemos recurrir a otras herramientas con el fin de asegurarnos la obtención de las soluciones, sin hacer uso de complejos y tediosos cálculos. En el artículo anterior (Resolución de las Ecuaciones en Diferencias. Vivat Academia $n^{\circ} 35$, mayo 2002) mostramos algunos de estos métodos de resolución. Asimismo, tratamos de familiarizar al lector con los teoremas de 
existencia y unicidad de las soluciones. Optamos por presentar el método del polinomio característico, también llamado del anulador, por ser el que mejor muestra el paralelismo de estas ecuaciones con las llamadas diferenciales. La presentación se realizó en casos particulares, haciendo uso de ejemplos simples de fácil comprensión, para después llegar a la generalización del método, por ser una forma sencilla de mostrar la teoría de las ecuaciones en diferencias. Sin faltar al rigor matemático, omitimos muchos de los lemas, teoremas y definiciones, habituales en estos casos, para evitar la dispersión de la idea principal, así como el excesivo volumen del trabajo, que harían oscuro al neófito la sencillez de este método.

En los precedentes trabajos, mencionamos que nuestro interés principal residía en la presentación de este tipo de ecuaciones con la intención de exponer finalmente la resolución numérica de ecuaciones diferenciales utilizando el método de las diferencias finitas, íntimamente ligado a las ecuaciones en diferencias estudiadas. Por consiguiente, este último artículo presenta, en primer lugar, el paralelismo entre ambos tipos de problemas, para finalizar estableciendo el llamado método de las diferencias finitas de resolución numérica de ecuaciones diferenciales, así como sus limitaciones.

\section{Paralelismo en la resolución de ecuaciones diferenciales y ecuaciones en diferencias}

Al igual que hemos procedido en los artículos precedentes, abordemos este apartado con el estudio de un caso particular muy simple de ecuación diferencial ordinaria de primer orden, no homogénea, con coeficientes constantes.

Sea una ecuación diferencial de la forma, (consideramos como variable dependiente el tiempo): 
$y^{\prime}-a y=b$

Un método de resolución muy socorrido, idéntico al del anulador para ecuaciones en diferencias, consiste en encontrar las soluciones del polinomio característico. Como se trata de una ecuación inhomogénea, debemos buscar primero la solución general de la parte homogénea, para después encontrar soluciones particulares de la ecuación completa. Así la ecuación homogénea asociada se escribirá:

$\mathbf{u}^{\prime}-\mathbf{a} \mathbf{u}=\mathbf{0}$

Su solución general se puede obtener utilizando el operador derivada, en este caso con respecto al tiempo, D (análogo al operador diferencia, de igual nomenclatura D, de las ecuaciones en diferencias), así como el operador identidad I. Por consiguiente, la ecuación homogénea anterior se podrá expresar en la forma:

$(\mathrm{D}-\mathrm{a} I) \mathrm{u}=\mathbf{0}$

Resolviendo el polinomio característico, equivalente a anular el paréntesis de la expresión anterior:

$P(r)=r-a=0=r=a$

que tiene una raíz real y simple, se concluye que la solución general de nuestra ecuación es:

$\mathbf{u h}=\mathbf{A} \cdot \mathbf{e r} \mathbf{t}$

es decir,

uh $=$ A ea $\mathbf{t}$ 
con A una constante a determinar posteriormente, haciendo uso de las condiciones iniciales.

El siguiente paso será obtener la solución particular yp de la ecuación completa, para lo que también podemos utilizar el anulador correspondiente. La ecuación diferencial puede escribirse en términos de operadores como:

$(D-a$ I) $y=b$

y dado que la derivada de una constante es nula, es decir $\mathbf{D} \mathbf{b}=\mathbf{0}$

$\mathrm{D} b=\mathrm{D}(\mathrm{D}-\mathrm{a} \mathrm{I}) \mathrm{y}=0$

Al igual que para las ecuaciones en diferencias, las raíces de los polinomios característicos de la ecuación anterior (anuladores), nos proporcionarán la solución (o soluciones) particular de la ecuación completa:

$\mathrm{D}(\mathrm{D}-\mathrm{a} \mathrm{I}) \mathrm{yp}=0$

Las raíces correspondientes son:

$\mathbf{D}=\mathbf{a}$

$\mathrm{D}=0$

Así, en este caso, una solución particular "yp" se encuentra entre las soluciones de la homogénea, es decir, $\mathbf{D}=\mathbf{2}$ es redundante y, por lo tanto, la solución particular de la ecuación completa será:

$y p=A \cdot e a t+B \cdot e t=A \cdot e 2 t+B$ 
con B otra constante a determinar. La solución completa será la suma de la solución general de la homogénea $\mathbf{u h}=\mathbf{A}$ ea $\mathbf{t}$ y la solución particular de la completa $\mathbf{y p} \mathbf{p} \mathbf{B}$. Esta suma ha de ser solución de la ecuación de partida

$y^{\prime}-a y=b$

Por consiguiente, obligando a verificarla:

$D(A \cdot e a t+B)-a(A \cdot a t+B)=b$

Recuérdese que D es el operador derivada. Por lo tanto:

$A$ a ea $t-a(A \cdot e a t+B)=b ; \grave{e ̀ ~} B=-b / a$

y, finalmente, se obtendrá la solución:

$B=-\frac{1}{2}$

$\mathrm{Si}$, además, tenemos condiciones iniciales, como p.e. $\mathrm{y}(0)=\mathrm{c}$, podría obtenerse el valor del coeficiente genérico "A" :

$y(0)=c=A$ ea $0-b / a=c=>A=b / a+c$

$y(t)=u h+y p=(b / a+c)$ ea $t-b / a$

Nótese que en las ecuaciones en diferencias se emplea el operador de cambio "E", para determinar el polinomio característico, y no el operador diferencia D. 


\section{Paralelismo funcional}

Normalmente una ecuación diferencial cualquiera, representa la relación entre una función $\mathrm{y}(\mathrm{x}, \mathrm{y}, \mathrm{z} \ldots)$ de las variables $\mathrm{x}, \mathrm{y}, \mathrm{z}, \ldots \mathrm{y}$ las correspondientes derivadas de la función respecto de dichas variables. En estos artículos pretendemos únicamente presentar una introducción a la resolución de ecuaciones diferenciales ordinarias por el método de las diferencias finitas, por ello nos centraremos en las ecuaciones de una sola variable y no estudiaremos los casos posibles de ecuaciones en derivadas parciales.

Consecuentemente, consideremos una función $\mathrm{y}(\mathrm{x})$, y sus posibles valores 'yi' en diferentes puntos ' $x i^{\prime}$ ', o lo que es lo mismo, para diferentes valores ' $x i$ ' de la variable $\mathrm{x}$, separados entre sí una distancia d.

Evidentemente, la representación gráfica de $\mathrm{y}(\mathrm{x})$ frente a $\mathrm{x}$ supone una curva, que puede aproximarse, en cada valor de la variable $\mathrm{x}$, por el correspondiente desarrollo en serie de Taylor. Ello significa que, conocido el valor de la función en el punto xi, podemos conocer el valor de esa misma función en el punto (xi + e), con e una cantidad muy pequeña, usando el concepto de derivada, en su primer orden y órdenes sucesivos. Así, si llamamos

yi al valor de $y(x i)$

$y i+1$, al valor de $y(x i+e)$

yi-1, al valor de $y(x i-e)$

$y i+2$, al valor de $y(x i+2 e)$ 
yi-2, al valor de $y(x i-2 e)$

y así sucesivamente,

podemos escribir, usando el citado desarrollo en serie de Taylor:

$y(x i+e)=y i+1=y i+e y^{\prime} i+$ términos de orden superior en e

donde por y'i entendemos el valor de la primera derivada de la función yi evaluada en el punto xi. Observado la anterior expresión nos daremos cuenta de que tenemos una ecuación en diferencias típica, con el valor de un estado en función del estado en un punto anterior, por medio de una función que es la primera derivada y un parámetro e. Así, en el caso de que la variable x sea el tiempo, tendremos el valor de la función como expresión de su valor en un instante anterior. A título de preparación al tratamiento numérico de las ecuaciones diferenciales, estableceremos, de una forma más sistemática, estas relaciones entre los valores secuenciales de una función y(xi) y sus derivadas calculadas en los puntos xi.

Consideremos, por tanto, un conjunto de valores de la función y(x),

$y i=y(x i)$, evaluados en puntos xi separados espacial y equidistantemente, siendo la separación "d". Utilizando el desarrollo en serie de Taylor, para los valores de la función en torno al punto xi, con yi el valor de la función evaluada en el punto xi obtendremos

$$
\begin{aligned}
& \mathrm{y}_{i+1}=\mathrm{y}_{\mathrm{i}} \pm \mathrm{d} \mathrm{y}_{\mathrm{i}}^{\prime}+\frac{\mathrm{d}^{2}}{2 !} \mathrm{y}_{\mathrm{i}}^{\prime \prime} \pm \frac{\mathrm{d}^{3}}{3 !} \mathrm{y}_{\mathrm{i}}^{\prime \prime \prime}+ \\
& + \text { términos de orden superior en } \mathrm{d}
\end{aligned}
$$


De aquí en adelante, denotaremos la derivada de orden n de la función yi evaluada en el punto xi en la forma yi(n).

De la expresión del desarrollo en serie de Taylor se pueden obtener tres aproximaciones para el valor de la derivada de orden $1,{ }^{y_{i}^{\prime}}$, de la función que nos ocupa. Para ello, en el primer caso, basta con restar las dos expresiones del desarrollo en serie, la de signo positivo y la de sigo negativo. En el segundo caso, se despeja de la ecuación con signo positivo el valor de la derivada y, en el tercero, se utiliza la ecuación con signo negativo para resolver la derivada de primer orden. Es decir:

$$
\begin{aligned}
& y_{i}^{(1)}=\frac{y_{i+1}-y_{i-1}}{2 d}-\frac{2 d^{3}}{2 d 3 !} y_{i}^{(3)}+\ldots \\
& y_{i}^{(1)}=\frac{y_{i+1}-y_{i}}{d}-\frac{d^{2}}{2 ! d} y_{i}^{(2)}+\ldots \\
& y_{i}^{(1)}=\frac{y_{i}-y_{i-1}}{d}+\frac{d^{2}}{2 ! d} y_{i}^{(2)}+\ldots
\end{aligned}
$$

La diferencia

$$
\mathrm{y}_{\mathrm{i}+1}{ }^{-\mathrm{y}_{\mathrm{i}-1}}
$$

$2 \mathrm{~d}$

es llamada aproximación central en diferencias a ${ }^{y_{i}^{(1)}}$. El error cometido al utilizar esta expresión es de un valor aproximado de (d2/6)yi(3) (salvo términos de orden superior en $\mathrm{d})$. 
La diferencia

$$
\text { yi+1 - yi }
$$

d

Es llamada aproximación hacia delante en diferencia a $y_{i}^{(1)}$. Es una aproximación más pobre que la anterior, ya que el error cometido al utilizar esta expresión es de un valor aproximado de (d/2)yi(2) (salvo términos de orden superior en $d$ ), siempre y cuando el valor de $d$ sea pequeño, por supuesto.

Análogamente, la diferencia

$$
\frac{y_{i-1}-y_{i}}{d}
$$

Es llamada aproximación hacia atrás en diferencia a ${ }^{y_{i}^{(1)}}$. El error cometido al utilizar esta expresión es de un valor aproximado igual al caso anterior, pero con signo invertido (salvo términos de orden superior en d) y, claramente, se trata de una aproximación peor que la aproximación central.

Podemos seguir calculando estas aproximaciones para las derivadas de orden superior de $\mathrm{y}(\mathrm{xi})$. Por ejemplo, mediante la suma de las expresiones con signo negativo y signo positivo, se consigue una aproximación central para yi(2):

$$
y_{i}^{(2)}=\left(\frac{d^{2} y}{d x^{2}}\right)_{x=x_{i}}=\frac{y_{i+1}-2 y_{i}-y_{i}-1}{d^{2}}+\ldots
$$

Esta aproximación, conocida como diferencia segunda de $\mathbf{y}(\mathbf{x})$, tiene un error aproximado de (d2/12)yi(4). 
Obviamente, si la función $\mathrm{y}(\mathrm{x})$ es un polinomio de la variable $\mathrm{x}$ suficientemente simple, a partir de un orden particular, todas las derivadas se anulan y no es necesario tener en cuenta los errores para obtener los valores de las derivadas a partir de las diferencias.

\section{El método de las diferencias finitas}

Vamos a presentar el método utilizando, como viene siendo habitual en este trabajo, un sencillo caso que dará al lector la pauta a seguir, con el menor número de dificultades posible. Consideremos, pues, una ecuación diferencial ordinaria de primer orden, típicamente representada por

$$
\frac{d y}{d x}=F(x, y)
$$

siendo x la variable independiente e y la variable dependiente. Si la ecuación puede resolverse analíticamente, no hay duda de que ese es el mejor método a adoptar. Cuando no se da esta posibilidad, debemos recurrir a las soluciones numéricas. El ejemplo que vamos a mostrar puede resolverse por métodos analíticos simples, sin embargo, para ilustrar la aproximación numérica es útil tener la solución analítica y comparar los resultados con los obtenidos por los métodos numéricos, siempre aproximados.

Un sencillo ejemplo de ecuación diferencial de la clase nombrada en el párrafo precedente y que tiene solución analítica puede ser

$y^{\prime}=-y$

Con la condición inicial $\mathbf{y}(\mathbf{0}) \mathbf{= 1}$. 
Utilizaremos una diferencia finita, de las definidas en el apartado anterior, para determinar de forma aproximada el valor de $y^{\prime}$. Si usamos la diferencia hacia delante tendremos

$$
\left(\frac{\mathrm{dy}}{\mathrm{dx}}\right)_{\mathrm{x}=\mathrm{xi}} \cong \frac{\mathrm{y}_{\mathrm{i}+1}-\mathrm{y}_{\mathrm{i}}}{\mathrm{d}}
$$

Por consiguiente, la relación de recurrencia, que nos convertirá la resolución de esta ecuación diferencial en la resolución de una típica ecuación en diferencias, será:

$$
\mathrm{y}_{\mathrm{i}+1}=\mathrm{y}_{\mathrm{i}}+\mathrm{d}\left(\frac{\mathrm{dy}}{\mathrm{dx}}\right)_{\mathrm{x}=\mathrm{xi}}=\mathrm{y}_{\mathrm{i}}-\mathrm{dy_{ \textrm {i } }}=(1-\mathrm{d}) \mathrm{y}_{\mathrm{i}}
$$

siendo "d" la separación entre dos puntos x consecutivos, es decir,

$y i+1=(1-d) y i$

$\mathrm{x} 0=0$

$\mathbf{x} \mathbf{1}=\mathbf{0}+\mathrm{d}$

$x 2=0+2 d$

$x 3=0+3 d$

y así sucesivamente.

Si partimos del valor cero para $x$, a fin de llegar al valor $x=0,5$, necesitamos $0,5=$ n.d pasos, o valoraciones sucesivas de la recurrencia, con un valor de $\mathrm{n}$ igual a 50, cuando elegimos para d el valor de 0,01, o lo que es lo mismo, debemos iterar 50 veces para determinar el valor de $\mathrm{y}(\mathrm{x}=0,5)$. Cuanto más pequeño sea el valor de $\mathrm{d}$ 
mayor será el número de pasos en la recurrencia pero, dado que el error cometido (ver la sección anterior) crece con alguna potencia de d, será tanto menor, cuanto menor sea d. Esta forma de proceder es conocida con el nombre de método de Euler. En nuestro caso concreto, dada la condición inicial $\mathbf{y}(\mathbf{0}) \mathbf{= 1}$, tendríamos para un valor de $\mathbf{d}=0,01$ :

$y(0)=1$

$y(0,01)=0,99$

$y(0,02)=0,99.0,99 \ldots$

$y(50)=y(x=0,5)=0,9950=(1-d) 50 y(0)$

En la siguiente tabla mostramos los valores de $\mathrm{y}(\mathrm{x})$, para la ecuación diferencial anterior, obtenidos usando diferentes valores de d y comparándolos con la solución exacta, posible a través de la resolución analítica de la ecuación que, para la condición inicial indicada, resulta ser:

$y(x)=\exp (-x)$ 
$\underline{\text { Tabla de los valores obtenidos para la solución de la ecuación diferencial }}$ $\mathrm{y}^{\prime}=-\mathrm{y}$, con diferentes valores del paso $\mathrm{d}$, utilizando el método de Euler $\mathrm{y}$ la diferencia hacia delante así como su comparación con los valores exactos de la solución

\begin{tabular}{|l|c|l|c|c|c|}
\hline \multirow{2}{*}{\begin{tabular}{c} 
Valores de \\
\cline { 1 - 5 }
\end{tabular}} & $\begin{array}{c}\text { Valores de } \\
\mathbf{x}\end{array}$ & $\begin{array}{c}\text { Valores de } \\
\mathbf{d}\end{array}$ & $\begin{array}{c}\text { Valores de } \\
\mathbf{d}\end{array}$ & $\begin{array}{c}\text { Valores de } \\
\mathbf{d}\end{array}$ & $\begin{array}{c}\text { Valores } \\
\text { exactos de } \\
\mathbf{y}(\mathbf{x})\end{array}$ \\
\cline { 2 - 5 } (condicín inicial) & 0,01 & 0,1 & 0,5 & 1 & 1 \\
\hline 0,5 & 0,605 & 0,590 & 0,500 & 0 & 0,607 \\
\hline 1 & 0,366 & 0,349 & 0,250 & 0 & 0,368 \\
\hline 1,5 & 0,221 & 0,206 & 0,125 & 0 & 0,223 \\
\hline 2 & 0,134 & 0,122 & 0,063 & 0 & 0,135 \\
\hline 2,5 & 0,081 & 0,072 & 0,032 & 0 & 0,082 \\
\hline
\end{tabular}

La observación de la tabla anterior da suficiente evidencia de que, para obtener una aproximación razonable, han de tomarse valores del paso d muy pequeños. En teoría se podrían tomar valores de d más grandes que la separación entre dos puntos sucesivos, pero, en estos casos, el resultado puede llegar a oscilar e incluso ser divergente. La ecuación asociada en diferencias que da lugar a la recurrencia es de la forma:

$y i+1=1$ yi

y una condición necesaria para la no divergencia es que el valor absoluto de 1 sea inferior a la unidad, es decir, $\mathrm{d}$ esté comprendido en el intervalo $0<\mathrm{d}<2$, aunque estos valores no aseguren la precisión. 
En parte, estas dificultades provienen de la pobre aproximación que representa la diferencia hacia delante. El valor de la función $\mathrm{y}(\mathrm{x})$ se evalúa en $\mathbf{x}=\mathbf{x i}+\mathrm{d} / \mathbf{2}$ para obtener la derivada en el punto $\mathrm{x}=$ xi. Podemos usar el llamado método de Milne, consistente en utilizar la aproximación central, que da menores valores para el error.

Algunas dificultades provienen de la propia forma de la ecuación diferencial y no de la aproximación utilizada.

\section{Conclusión}

Con esta entrega damos por terminado el trabajo sobre las ecuaciones en diferencias y su aplicación a la resolución numérica de ecuaciones diferenciales. No hemos descrito otras posibles formas de encontrar soluciones a las ecuaciones en diferencias, lo que dejamos para posibles trabajos futuros, porque es concretamente el método del anulador el que marca con más intensidad el paralelismo entre las ecuaciones en diferencias y las diferenciales, que era nuestro objetivo principal. Tampoco vamos a mencionar otros métodos numéricos que harían excesivamente largo el trabajo.

En el presente artículo hemos mostrado dichas analogías y hemos establecido el llamado método de las diferencias finitas de resolución numérica de ecuaciones diferenciales, así como sus limitaciones, que podemos resumir en:

1) Las aproximaciones dan resultados muy imprecisos en los valores de las derivadas, salvo que se elijan pasos muy pequeños, haciendo el cálculo excesivamente largo en el tiempo.

2) Los errores de redondeo pueden conducir a inestabilidades de la solución numérica (bien oscilaciones, bien divergencias). 


\section{Bibliografía}

DEWNEY, A. K. (1985): "Juegos de ordenador" en Revista Investigación y Ciencia, no 100 de Enero de 1985.

ELAYDI, S. N. (1999): An Introduccion to Difference Equations. Springer.

RILEY, K. F.; HOBSON, M. P. y VENCE, S. J. (1997): Mathematical Methods for Phisics and Engineering. Cambridge University Press. 